\title{
Estimación de la ubicación relativa de una cámara utilizando un sistema de visión monocular
}

\section{Estimation of relative location of a camera using a monocular vision system}

\author{
MARTÍNEZ-DÍAZ, Saúl†* \\ Instituto Tecnológico de la Paz / Tecnológico Nacional de México División de estudios de Posgrado e Investigación
}

ID $1^{\text {er }}$ Autor: Saúl, Martínez-Díaz / ORC ID: 0000-0003-4962-5995, Researcher ID Thomson: Q-7112-2019, CVU CONACYT ID: 175255

\section{Resumen}

Objetivo: Estimar la ubicación de una cámara con respecto a objetos en el mundo real, utilizando visión monocular. Metodología: En este artículo se presenta un método para calcular la ubicación relativa de la cámara con respecto a un grupo de puntos ubicados en el espacio tridimensional. El método requiere únicamente tres puntos de referencia fijos, de los cuales se debe conocer la distancia real entre cada par de puntos. Con esta información es posible estimar la ubicación relativa de la cámara al ser desplazada, utilizando imágenes sucesivas que contengan los mismos puntos. Contribución: En los últimos años el poder de procesamiento de las computadoras ha crecido considerablemente $\mathrm{y}$, con ello, también ha aumentado el interés de la comunidad científica por la odometría visual. Para este propósito, en muchos casos, es conveniente utilizar una sola cámara. Desafortunadamente, un sistema monocular permite estimar la ubicación de la cámara con respecto a algún objeto del mundo real únicamente hasta un factor de escala. La principal contribución de este trabajo es la estimación de la ubicación de la cámara en coordenadas del mundo real con respecto a un objeto de referencia.

Visión artificial, Odometría visual, Sistema monocular

\begin{abstract}
Objective: Estimate the location of a camera with respect to objects in the real world, using monocular vision. Methodology: In this paper we introduce a method to calculate the relative location of the camera with respect to a group of points located in the three-dimensional space. The method requires only three fixed reference points of which the real distance between each pair of points must be known. With this information it is possible to estimate the relative location of the camera when it is moved, using successive images that contain the same points. Contribution: In recent years, processing power of computers has grown considerably and, with this, the interest of the scientific community in visual odometry has also increased. For this purpose, in many cases, it is convenient to use a single camera (monocular system). Unfortunately, a monocular system allows to estimate the location of the camera with respect to some object in the real world only up to a scale factor. The main contribution of this work is the estimation of the location of the camera in real world coordinates with respect to a reference object.
\end{abstract}

\author{
Artificial vision, Visual odometry, Monocular \\ system
}

Citación: MARTÍNEZ-DÍAZ, Saúl. Estimación de la ubicación relativa de una cámara utilizando un sistema de visión monocular. Revista de Ingeniería Innovativa. 2019. 3-10: 18-23

\footnotetext{
*Correspondencia al Autor (Correo electrónico: saulmd@itlp.edu.mx)

$\dagger$ Investigador contribuyendo como primer Autor.
} 


\section{Introducción}

En años recientes, debido al incremento en la capacidad de procesamiento de las computadoras modernas y a la reducción de su costo, ha sido posible desarrollar aplicaciones de visión artificial que respondan en tiempo real. En ese sentido, una de las áreas que han generado mayor interés es la odometría visual. Esto se debe a que existen muchas situaciones en las que se puede utilizar esta tecnología. Entre ellas se encuentran la navegación de robots autónomos como en los trabajos de (Wang, 2015) y el de (Tumer, 2011), realidad aumentada como se explica en (Chatzopoulos, 2017) y la inspección en sistemas industriales como en (Xu, 2016) y (Shanmugamani, 2014).

En estos casos se requiere información de la ubicación de los objetos en coordenadas tridimensionales del mundo real. En algunas aplicaciones se utilizan sistemas GPS, sin embargo, estos son susceptibles a interferencias, sobre todo en lugares rodeados de edificios, bajo puentes $\mathrm{o}$ en interiores. Además, presentan márgenes de error grandes, de hasta varios decímetros. Para determinar la ubicación relativa también se pueden utilizar las cámaras RGBD, pero además de su alto costo, utilizan sensores infrarrojos para determinar la profundidad a la que se encuentran los objetos, lo cual dificulta e incluso impide su aplicación en algunos lugares iluminados con luz natural. Por otro lado, los sistemas de visión son de bajo costo $\mathrm{y}$, en algunos casos, presentan ventajas con respecto a las tecnologías dominantes que se utilizan en la actualidad.

Un sistema de visión puede estar compuesto por una sola cámara, en cuyo caso se denomina monocular, o por dos o más cámaras, denominado estereoscópico. En los sistemas estereoscópicos, por lo general, es necesario realizar obtener la rotación y traslación de una cámara con respecto a la otra, por medio de un procedimiento de calibración. Los parámetros obtenidos en la calibración se deben mantener fijos para estimar la ubicación de los objetos del mundo real. Para calcular la posición de un punto en el espacio tridimensional, cada cámara debe capturar una imagen que contenga dicho punto, después debe identificar las coordenadas del punto dentro de las dos imágenes y triangular para obtener sus coordenadas tridimensionales.
Sin embargo, los sistemas estereoscópicos presentan también algunos inconvenientes prácticos. Por ejemplo, cada cámara suele dar diferente respuesta a una misma señal de entrada, lo cual dificulta el proceso de identificación; También, requiere mayor espacio físico, el consumo de energía es mayor, el costo computacional para procesar dos imágenes en cada ocasión es mayor y la calibración de las cámaras se puede perder debido a movimientos o vibraciones. Además, cuando se observan puntos lejanos, el sistema se degenera $y$ tiende a comportarse como monocular.

Por todo lo anterior, los sistemas monoculares se presentan como una buena alternativa. Uno de los principales problemas en un sistema monocular es que, con una sola imagen, la ubicación de algún punto en el espacio tridimensional se puede conocer únicamente hasta cierto factor de escala. Este factor se puede calcular si se conoce la profundidad de dicho punto. En la literatura se han propuesto varias aproximaciones para estimar este factor de escala: En (Tarrio, 2017) utilizan una cámara como sensor principal y una unidad de medición inercial para determinar la escala.

En (Yin, 2017) estiman la profundidad utilizando una red neuronal convolucional, esta estimación se refina entrenando la red con las imágenes consecutivas. En (Said, 2012) utilizan un patrón de tres círculos concéntricos de diámetro conocido en un plano con la cámara perpendicular a dicho plano para calcular la profundidad inicial. En (Yao, 2017) suponen que el campo de visión de la cámara montada en un dirigible es siempre perpendicular a la tierra, se coloca la cámara a una distancia conocida, se detecta el rostro de una persona centrado en la imagen, se cuentan los pixeles y con estos datos se establece una relación para calcular la profundidad, siempre que se detecte el mismo rostro en futuras imágenes.

En este trabajo se presenta un método para calcular la profundidad de un grupo de tres puntos fijos en el espacio tridimensional, utilizando una cámara calibrada de bajo costo. Para ello se debe conocer únicamente la distancia entre cada par de puntos. 
Los puntos pueden estar en cualquier posición y no se requiere que la cámara esté perpendicular al plano formado por los tres puntos, como en otras propuestas. Capturando con la misma cámara al menos dos imágenes que contengan los mismos puntos en diferente pose, se calcula la ubicación relativa actual de la cámara. El resto del artículo se organiza de la siguiente manera: la sección 2 presenta los conceptos básicos que soportan el trabajo, en la sección 3 se presenta el método propuesto, en la sección 4 se presentan algunos resultados experimentales y en la sección 5 se resumen las conclusiones.

\section{Conceptos Básicos}

\section{Modelo Ojo de Aguja}

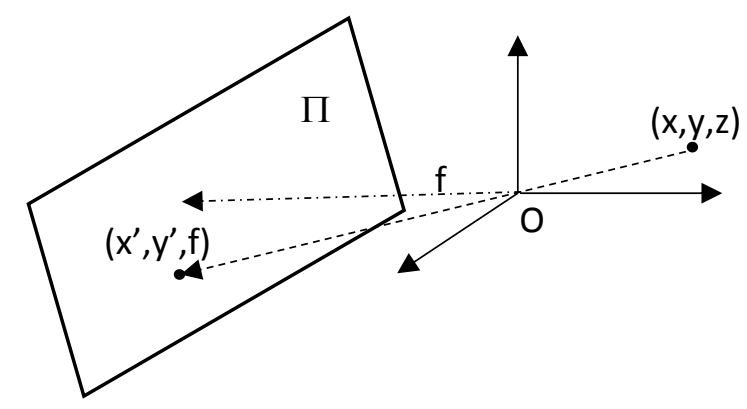

Figura 1 Modelo ojo de aguja Fuente: Elaboración Propia

El modelo ojo de aguja es utilizado ampliamente para representar la formación de imágenes en una cámara. En la figura 1 se muestra el principio básico de este modelo. Como se puede apreciar, cada punto con coordenadas (x, y, z) en el espacio tridimensional es proyectado a través del ojo de aguja al punto (x', y', f), con coordenadas en pixeles, sobre el plano $\Pi$ de la cámara. En esta figura, f es la distancia focal de la cámara, la cual se puede obtener mediante el proceso de calibración. En consecuencia, por semejanza de triángulos, se puede establecer que (BarrancoGutiérrez, 2018):

$\frac{x \prime}{x}=\frac{y \prime}{y}=\frac{f}{z}=\lambda$

Donde $\lambda$ es un factor de escala. Cuando dicho factor es conocido, esto permite calcular las coordenadas tridimensionales. Además, se requiere conocer las coordenadas del punto dentro de la imagen y la distancia focal.

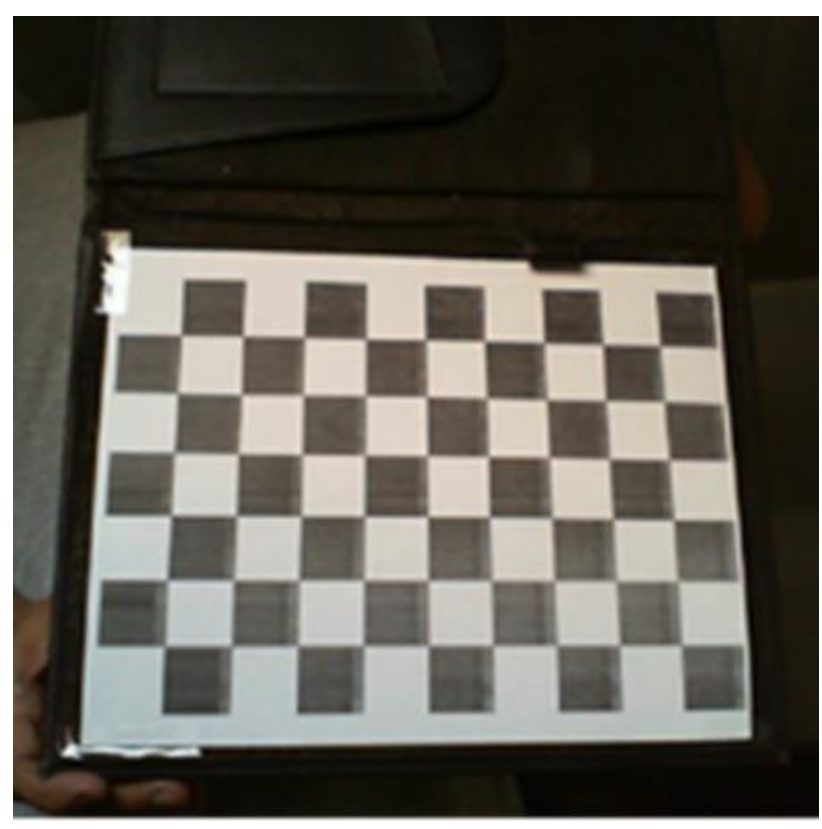

Figura 2 Patrón de calibración

Fuente: Elaboración Propia

\section{Calibración de la cámara}

Existen varios métodos para llevar a cabo la calibración de la cámara. Uno de los más populares es el propuesto por (Zhang, 2000), el cual está basado en el modelo ojo de aguja. Con esta técnica de calibración solamente se requiere que la cámara observe un patrón plano (ver figura 2) tomado desde diferentes orientaciones. El patrón o la cámara pueden ser movidos libremente y no es necesario conocer el movimiento realizado. Esta calibración permite obtener los parámetros intrínsecos y extrínsecos de la cámara, los cuales se relacionan de la siguiente forma (Hartley, 2004):

$\lambda \mathbf{P}^{\prime}=\mathbf{A}[\mathbf{R} \mathbf{t}] \mathbf{P}$

Donde $\mathbf{P}$ es un punto del espacio tridimensional, representado en coordenadas homogéneas; P' es la proyección del mismo punto en el plano de la cámara, representado en coordenadas bidimensionales homogéneas; [R t] es una matriz de parámetros extrínsecos (rotación y traslación); $\lambda$ es un factor de escala arbitrario y A es la matriz de parámetros intrínsecos. La matriz de parámetros intrínsecos está definida por:

$\mathbf{A}=\left[\begin{array}{ccc}f_{x} & 0 & c_{x} \\ 0 & f_{y} & c_{y} \\ 0 & 0 & 1\end{array}\right]$ 
Aquí $f_{x}, f_{y}$ proporcionan información (dependiendo del tamaño del pixel) de la distancia focal en la dirección de $x$ e $y$, respectivamente; $c_{x}, c_{y}$ son las coordenadas del punto principal de la imagen.

\section{Métodos}

\section{Cálculo de profundidad}

De la ecuación (1) se pueden obtener las siguientes relaciones:

$x=\frac{x^{\prime}}{f} z$

$y=\frac{x y^{\prime}}{f} z$

Suponiendo que se tienen al menos tres puntos en el espacio tridimensional con coordenadas $\left(x_{1}, y_{1}, z_{1}\right),\left(x_{2}, y_{2}, z_{2}\right), \mathrm{y}\left(x_{3}, y_{3}, z_{3}\right)$, respectivamente. Suponiendo también que las distancias $d_{1}, d_{2}$ y $d_{3}$ entre cada par de puntos son conocidas. Utilizando las relaciones en las ecuaciones (4) y (5), la distancia euclidiana cuadrática entre cada par de puntos se puede calcular por:

$d_{1}=\left(\frac{x 1_{1}}{f} z_{1}-\frac{x \prime_{2}}{f} z_{2}\right)^{2}+\left(\frac{y \prime_{1}}{f} z_{1}-\frac{y \prime_{2}}{f} z_{2}\right)^{2}+\left(z_{1}-z_{2}\right)^{2}$
$d_{2}=\left(\frac{x \prime_{1}}{f} z_{1}-\frac{x t_{3}}{f} z_{3}\right)^{2}+\left(\frac{y \prime_{1}}{f} z_{1}-\frac{y \prime_{3}}{f} z_{3}\right)^{2}+\left(z_{1}-z_{3}\right)^{2}$
$d_{3}=\left(\frac{x \prime_{3}}{f} z_{3}-\frac{x z_{2}}{f} z_{2}\right)^{2}+\left(\frac{y \prime_{3}}{f} z_{3}-\frac{y t_{2}}{f} z_{2}\right)^{2}+\left(z_{3}-z_{2}\right)^{2}$

Este sistema de ecuaciones se puede resolver para las $z_{i}$, las cuales representan la profundidad a la que se encuentra cada punto con respecto a la cámara. Sustituyendo estos resultados en las ecuaciones (4) y (5) es posible calcular también los valores de las $x_{i}$ y $y_{i}$, para obtener las coordenadas tridimensionales de los tres puntos.

\section{Estimación de Ubicación Relativa}

Una vez que se obtienen las coordenadas tridimensionales de al menos tres puntos, es posible obtener la ubicación relativa de la cámara al desplazarse. Esto es posible si la cámara observa los mismos tres puntos, calculando la transformación rígida entre ellos.
Una transformación rígida es una transformación geométrica que preserva la distancia euclidiana entre cada par de puntos, y se puede obtener mediante la descomposición en valores singulares (SVD, por sus siglas en inglés) de la matriz de covarianza.

Formalmente, dada una matriz cuadrada A de $m \times m$, la SVD descompone dicha matriz de la siguiente forma (Bradski, 2008):

$$
\mathbf{A}=\mathbf{U} \cdot \mathbf{W} \cdot \mathbf{V}^{\mathrm{T}}
$$

Donde $\mathbf{U}$ y $\mathbf{V}$ son matrices de $m \times m$ y $n \times n$, respectivamente y $\mathbf{W}$ es una matriz diagonal de $m \times n$. Con este proceso aplicado a cada nueva imagen, se puede conocer la ubicación relativa de la cámara en cada instante de tiempo.

\section{Algoritmo básico}

El algoritmo básico aplicado a cada imagen es:

1. Capturar una imagen conteniendo el objeto de referencia

2. Convertir la imagen a escala de grises

3. Corregir la distorsión radial (Bradski, 2008)

4. Detectar esquinas del patrón utilizando el método de Harris (Harris, 1988)

5. Seleccionar tres esquinas del objeto de referencia, cuyas distancias sean conocidas

6. Resolver el conjunto de ecuaciones (6) con el algoritmo de descenso de gradiente propuesto en (Moré, 1983)

\section{Resultados}

Para probar el método propuesto se utilizó el patrón de calibración mostrado en la figura 2 . Cada cuadro es de 27×27 milímetros. Se utilizó una cámara USB Microsoft configurada para entregar imágenes en RGB con una resolución de 640x480x3 pixeles. La cámara se calibró utilizando el método de Zhang (Zhang, 2000).

La cámara fue colocada a diferentes distancias del patrón y se aplicó el algoritmo propuesto. En la práctica, aun cuando se tomen dos imágenes con la misma cámara y desde la misma pose, el resultado no es idéntico debido a factores como ruido aleatorio en los sensores y variaciones en la fuente de iluminación. 
Por esta razón, para garantizar que los resultados sean estadísticamente correctos se efectuaron 30 pruebas en cada posición. Los valores obtenidos del promedio $\mathbf{m}$ y la desviación estándar $\sigma$ y los valores medidos se muestran en la tabla 1; todas las medidas están en milímetros.

\begin{tabular}{|l|c|c|}
\hline Distancia medida & $\mathbf{m}$ & $\boldsymbol{\sigma}$ \\
\hline 400 & 400.55 & 0.59 \\
\hline 450 & 449.51 & 0.71 \\
\hline 500 & 500.31 & 0.79 \\
\hline 550 & 550.63 & 0.96 \\
\hline 600 & 600.96 & 0.98 \\
\hline
\end{tabular}

Tabla 1 Profundidad medida vs calculada Fuente: Elaboración Propia

Como se puede apreciar en la tabla, la desviación estándar, es menor a $0.25 \%$ del promedio, en todos los casos. Esto indica que la incertidumbre de la medición es muy baja, con un error máximo del orden de un par de milímetros.

Posteriormente, se probó el cálculo de la ubicación relativa de la cámara en el espacio tridimensional. Para ello se utilizó un dispositivo Kinect 2, con el propósito de comparar los resultados. Kinect es un sensor tipo RGBD, el cual cuenta con una cámara a de luz visible con una resolución de 1920x1080x3 pixeles y un sistema infrarrojo que provee información de la profundidad de los objetos. El experimento consistió en desplazar la cámara a diferentes posiciones y calcular su ubicación con el método propuesto. Con ayuda del Toolbox de MATLAB para Kinect se obtuvieron las coordenadas de la cámara en cada posición.

El gráfico 1 muestra los resultados obtenidos de la comparación entre los valores calculados y los obtenidos con Kinect; para mayor claridad, con las tres coordenadas se calculó únicamente un valor, utilizando la fórmula de la distancia euclidiana. El coeficiente de correlación entre ambas gráficas es del $98.4 \%$, lo cual indica que los resultados son casi idénticos.

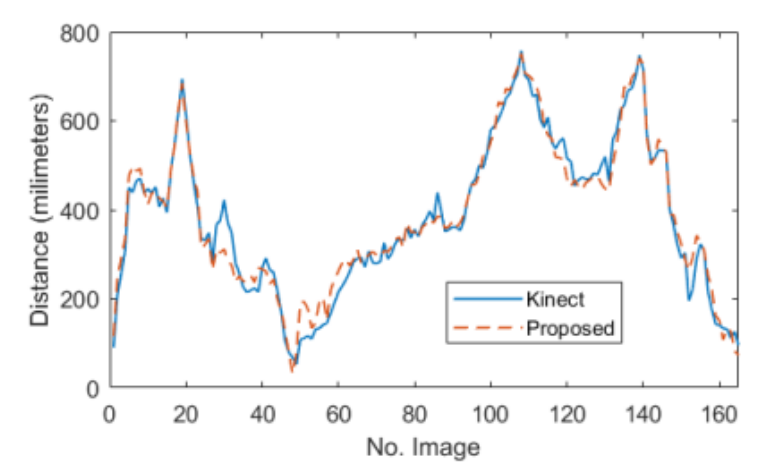

Gráfico 1 Comparación de cálculo de ubicación entre Kinect y el método propuesto

Fuente Elaboración Propia

\section{Conclusiones}

En este artículo se presentó un método para calcular la ubicación relativa de una cámara con respecto a al menos tres puntos de referencia en el espacio tridimensional utilizando un sistema de visión monocular. Para el cálculo solamente es necesario conocer la distancia entre cada par de puntos. Esta información puede utilizarse en sistemas de control industrial, realidad aumentada o navegación de robots autónomos, que utilicen una sola cámara.

Los resultados experimentales muestran un buen desempeño del algoritmo en imágenes tomadas con una cámara de bajo costo en condiciones no controladas. El cálculo de las coordenadas tridimensionales comparado contra un sistema RGBD comercial fue satisfactorio. La ventaja del método propuesto es que requiere únicamente hardware de bajo costo y es menos restrictivo y puede aplicarse en una mayor variedad de escenarios. Por lo anterior, consideramos que el trabajo es una contribución al avance científico en el área de la visión por computadora, en particular en el tema de odometría visual monocular.

\section{Agradecimiento}

Este proyecto fue financiado por el Tecnológico Nacional de México - Instituto Tecnológico de La Paz y el Consejo Nacional de Ciencia y Tecnología.

\section{Referencias}

Barranco-Gutiérrez, M.-D. G.-T. (2018). Visión Estereoscópica con MATLAB y OPENCV. La Paz: Pearson. 
Bradski, K. (2008). Learning OpenCV. Sebastopol, California: O'Reilly.

Chatzopoulos, B. H. (2017). Mobile augmented reality survey: from where we are to where we go. IEEE Access, 69176950.

Harris, S. (1988). A combined corner and edge detector . Proceedings of the 4th Alvey Vision Conference, (pp. 147-151).

Hartley, Z. (2004). Multiple View Geometry in Computer Vision . Cambridge University Press. Moré, S. (1983). Computing a trust region step. Journal on Scientific and Statistical Computing, 553-572.

Said, S. W. (2012). Depth estimation for a mobile platform using monocular vision. International Symposium on Robotics and Intelligent Sensors 2012 (IRIS 2012), (págs. 945950).

Shanmugamani, S. R. (2014). Detection and classification of surface defects of gun barrels using computer vision and machine learning. Measurement, 222-230.

Tarrio, P. (2017). Realtime edge based visual inertial odometry for MAV teleoperation in indoor environments. J. Intell. Robot Syst, 1-18. Tumer, K. y. (2011). Adaptive navigation for autonomous robots. Robotics and Autonomous Systems, 410-420.

Wang, L. L. (2015). Vision-based tracking control of underactuated water surface robots without direct position measurement. IEEE Transactions on Control Systems Technology, 2391-2399.

Xu, H. L. (2016). A machine-vision inspection system for conveying attitudes of columnar objects in packing processes. Measurement, 255-273.

Yao, A. T. (2017). Monocular vision-based human following on miniature robotic blimp. 2017 IEEE International Conference on Robotics and Automation (ICRA), (págs. 32443249).
Yin, W. D. (2017). Scale recovery for monocular visual odometry using depth estimated with deep convolutional neural fields. 2017 IEEE International Conference on Computer Vision (pp. 5871-5879). IEEE.

Zhang. (2000). A flexible new technique for camera calibration. IEEE Transactions on Pattern Analysis and Machine Intelligence, 1330-1334. 